OPEN ACCESS

Edited by:

Leonid Perlovsky,

Harvard University and Air Force Research Laboratory, United States

Reviewed by: Shinya Fujii, Keio University, Japan Enrico Glerean, Aalto University, Finland

*Correspondence: Andrea Ravignani andrea.ravignani@gmail.com

Specialty section:

This article was submitted to Auditory Cognitive Neuroscience,

a section of the journal

Frontiers in Psychology

Received: 25 May 2017 Accepted: 30 September 2017 Published: 06 November 2017

Citation:

Ravignani A and Madison G (2017) The Paradox of Isochrony in the Evolution of Human Rhythm.

Front. Psychol. 8:1820.

doi: 10.3389/fpsyg.2017.01820

\section{The Paradox of Isochrony in the Evolution of Human Rhythm}

\author{
Andrea Ravignani ${ }^{1,2,3 *}$ and Guy Madison ${ }^{4}$ \\ ${ }^{1}$ Language and Cognition Department, Max Planck Institute for Psycholinguistics, Nijmegen, Netherlands, ${ }^{2}$ Veterinary and \\ Research Department, Sealcentre Pieterburen, Pieterburen, Netherlands, ${ }^{3}$ Artificial Intelligence Lab, Vrije Universiteit Brussel, \\ Brussels, Belgium, ${ }^{4}$ Department of Psychology, Umeå University, Umeå, Sweden
}

Isochrony is crucial to the rhythm of human music. Some neural, behavioral and anatomical traits underlying rhythm perception and production are shared with a broad range of species. These may either have a common evolutionary origin, or have evolved into similar traits under different evolutionary pressures. Other traits underlying rhythm are rare across species, only found in humans and few other animals. Isochrony, or stable periodicity, is common to most human music, but isochronous behaviors are also found in many species. It appears paradoxical that humans are particularly good at producing and perceiving isochronous patterns, although this ability does not conceivably confer any evolutionary advantage to modern humans. This article will attempt to solve this conundrum. To this end, we define the concept of isochrony from the present functional perspective of physiology, cognitive neuroscience, signal processing, and interactive behavior, and review available evidence on isochrony in the signals of humans and other animals. We then attempt to resolve the paradox of isochrony by expanding an evolutionary hypothesis about the function that isochronous behavior may have had in early hominids. Finally, we propose avenues for empirical research to examine this hypothesis and to understand the evolutionary origin of isochrony in general.

\section{Keywords: synchrony, prediction, interaction, coordination, turn-taking, evolution of music, evolution of speech}

\section{CONTENTS}

This paper deals with isochronous temporal patterns. The emphasis is on the quantitative properties of isochronous patterns, and their perception and production in humans. The paper is organized in seven sections, namely:

(1) What is isochrony?, where we lay out crucial definitions and summarize basic relevant concepts;

(2) The relevance of isochrony to human music and speech, where we discuss how isochrony might partly underlie some behaviors in modern humans, such as music, speech and dance;

(3) Mathematics, physics and signal processing, where we discuss isochrony from the pure perspective of its physical and mathematical structure (as opposed, for instance, to its biological, behavioral or cognitive nature);

(4) Physiology and neuroscience, where we suggest how isochronous patterns have biological and psychological relevance for living organisms;

(5) Comparative cognition: Non-human animals, where we briefly summarize previous empirical attempts in finding, either directly or indirectly, isochronous behaviors in other species; 
(6) Isochrony in interaction, where we move from isochronous behaviors in single individuals to group behaviors potentially involving isochrony;

(7) Evolutionary hypotheses and future empirical work, where we join all strands laid out in the previous six sections, and sketch an evolutionary account for the origin of isochrony in our species.

The aim of this paper is not to provide an exhaustive review of each of these areas. Rather, we attempt to establish a first connection between as many explanatory levels of isochrony as possible, across scientific disciplines and research traditions.

\section{WHAT IS ISOCHRONY?}

Music is a complex phenomenon composed of interdependent parts. While a holistic approach is always important, the analytic, scientific method works by first analyzing constituent components individually (Fitch, 2015). Rhythm is a crucial dimension of human music. In common language, but also in scientific publications, different meanings are often conflated into the word 'rhythm' (see Table 1 for definitions). In the most general definition, rhythm denotes a pattern of events in time (McAuley, 2010). An isochronous pattern is a rhythm where all intervals between events are equal, like those of a metronome. Hence, all isochronous sequences are rhythmic, but not viceversa (Figure 1). A third related concept is the beat, namely the psychological tendency to superimpose an isochronous grid to a rhythmic sequence (a.k.a. pulse or beat perception, Figure 2C). The focus of the present paper is the evolutionary significance of the human perception and production of rhythmic sequences that are physically isochronous, henceforth simply 'isochronous.' We deliberately avoid discussing pulse and beat perception, as these have been object of much empirical research and many theoretical frameworks. Pure isochrony has received comparatively less attention.

Humans are particularly good at producing and perceiving both rhythmic and isochronous patterns (Bolton, 1894; Fitch, 2009, 2015; Motz et al., 2013; Ravignani et al., 2017). Yet, while general rhythm capacities could be biologically useful for foraging, mating, navigating the environment and predicting events, propensity for isochrony does not seem to confer any evolutionary advantage to modern humans (Fitch, 2009). Humans' aptitude for isochrony contrasted with its apparent lack of evolutionary function constitutes the paradox of isochrony. Below we offer some perspectives on the presence of isochrony in nature and in modern humans' everyday life.

Humans have extraordinary abilities to deal with isochronous behaviors. We can detect deviations from isochrony on the order of $20 \mathrm{~ms}$ or $4 \%$ of the interval to be timed, but we can also perceive the underlying isochrony even in the face of random deviations and gradual increases/decreases in intervals (Bolton, 1894; Madison and Merker, 2002, 2004; Max and Yudman, 2003; Repp, 2005; Madison, 2009; Motz et al., 2013; Repp and $\mathrm{Su}, 2013)$. When confronted with isochronous sequences where intervals have been slightly jittered, humans will tend

TABLE 1 | Definitions of key concepts discussed in the paper (Expanded and modified from Ravignani and Norton, 2017).

\begin{tabular}{|c|c|}
\hline Name & Definition \\
\hline Rhythm & Pattern of events in time; i.e., 'a specific succession of durations' (Wade, 2004, p. 57). \\
\hline Interval & Temporal duration encompassed by two events. \\
\hline Idealized isochrony & A rhythmic pattern where all intervals have equal duration. \\
\hline Isochrony (empirical) & $\begin{array}{l}\text { A rhythmic pattern where all intervals have roughly equal duration. Alternatively, a rhythmic pattern obtained by jittering events in an idealized } \\
\text { isochronous pattern. }\end{array}$ \\
\hline Anisocronous & Not isochronous. Operationally, a pattern exhibiting less isochrony than another pattern under consideration. \\
\hline Pulse a.k.a. beat & $\begin{array}{l}\text { Psychological tendency to superimpose an isochronous grid (not necessarily present in the physical signal) to a rhythmic sequence. In some } \\
\text { cases, such as here, pulse and beat are defined as equivalent. In some other cases, the beat is defined as the isochronous grid generated via } \\
\text { metrical expectations (see below). }\end{array}$ \\
\hline Synchronous & Two rhythmic patterns where each event in one pattern occurs at the same time as a corresponding event in the other pattern. \\
\hline Meter & Hierarchical organization of temporal events based on stress and other spectral properties, such as loudness alternation, pitch variation, etc. \\
\hline Syllable-timed & Language where all accented and unaccented syllables are isochronous. \\
\hline Stress-timed & Language where all stressed syllables occur isochronously. \\
\hline Mora-timed & Language where all moras, consisting in syllables or some syllable combinations, are isochronous. \\
\hline Endogenous & Pattern production involving a 'self-sustained clock that governs the rhythm' (Pikovsky et al., 2003, p. 87). \\
\hline Exogenous & Pattern production that is influenced by an external oscillator/clock/pacemaker. \\
\hline Reactive & Exogenous mechanism of production of a temporal interval whose duration is a direct response from a previous duration in the environment. \\
\hline Predictive & $\begin{array}{l}\text { Exogenous mechanism of production of a temporal interval whose duration is based on expectations about an upcoming environmental } \\
\text { duration. }\end{array}$ \\
\hline Entropy & $\begin{array}{l}\text { The expected value of the information content of a signaling system. It quantifies the amount of information in a signal, without any reference to } \\
\text { its meaning. }\end{array}$ \\
\hline Redundancy & $\begin{array}{l}\text { A measure of the actual information content relative to the maximum possible information content of a signaling system. High redundancy } \\
\text { means that information is more 'diluted.' }\end{array}$ \\
\hline Compressibility & Here: inverse of entropy. \\
\hline Expressivity & Capacity of a signaling system to convey different meanings. \\
\hline
\end{tabular}




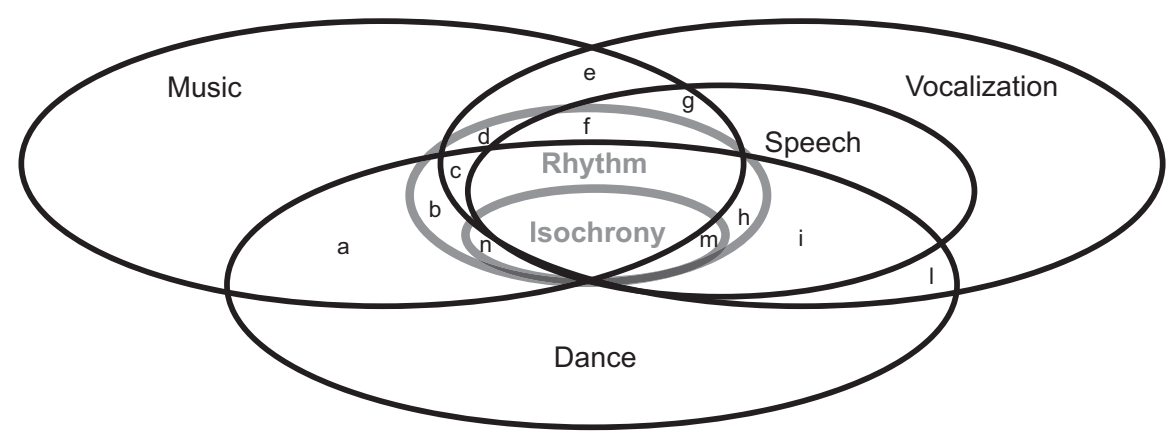

FIGURE 1 | Conceptual relationships among music, vocalizations, speech, and dance with respect to temporal structure, rhythm, and isochrony. Lowercase letters a- $n$ denote some possible intersections. For instance, $g$ denotes shared timing between speech and music (but not dance), while $n$ denotes isochronous music and dance with no commonalities with speech. It is up to empirical research to decide which ones of these are empty. Notice that the question of intersections is not moot: one of the first papers on the origins of dance (Laland et al., 2016), for instance, implicitly suggested that the evolutionary path for the emergence of dance had to go from e to $m$ to $h$.

to regularize the intervals and perceive the whole sequence as isochronous (for psychophysical thresholds see Friberg and Sundberg, 1995; Madison and Merker, 2002; Merker et al., 2009). Also when producing isochronous sequences the variability is around $4 \%$, but it can be decreased to about $2 \%$ of the interval when synchronizing to a sound sequence with faster metrical levels (Madison, 2014). Listening to an isochronous sequence typically induces a beat (Figure 2C), which in turn generates expectations that future events will fall into a multiple or integer subdivision of the beat. A violation of the expectation, such as slightly changing the onset of one event, can be measured by mismatch negativity (Motz et al., 2013). The processes underlying this reaction are subliminal (Madison and Merker, 2004) and require no learning, which indicates that the beat is a very basic, inherited phenomenon.

In evolutionary biology, a behavioral trait can appear for a number of reasons. It can be an evolutionary adaptation, namely a trait which evolved to increase a species' fitness in a given environment. As such, it may more or less have lost its adaptive value due to changes in the environment, while still prevailing in the population because it has not been selected against. It can also be a by-product of other evolutionary processes, a so-called exaptation. As such, isochrony might have been recruited for purposes unrelated to the pressures which caused its early emergence (Merker et al., 2009). In the present context, isochrony refers to humans' perception and production of isochronous event sequences within the bounds and constraints reviewed above.

\section{THE RELEVANCE OF ISOCHRONY TO HUMAN MUSIC AND SPEECH}

The roots of the human propensity for isochrony are clearly found in our biology, specifically in some preparedness of our neural system (Buzsaki, 2006; Arnal and Giraud, 2012; Fujioka et al., 2012; Fujii and Wan, 2014; Merchant et al., 2015). For example, newborn babies react differently to isochronous than to anisochronous sequences (Honing et al., 2009). Similarly, children aged 2-4 years show motoric isochronous behavior with clear periodicities, though little tempo adjustment (Eerola et al., 2006). Although isochrony in music is a human universal (Figure 2C), there is considerable variation across the worlds' music cultures. Western musical cultures appear to employ isochrony most thoroughgoingly, for example with rhythmic sequences being composed of isochronous subsequences (e.g., Bach). When rhythmic patterns are not isochronous, they are based on a psychologically induced sense of beat (Merker et al., 2009). In these cases, notes that are played continuously confirm or violate the induced pulse (Figure 2D), either in a structural or expressive manner (see Merker, 2014 for a novel perspective). African music is also isochronous at some descriptive level (though see Figure 3), while Asian music tends to be less so. Some North-American Indian, Javanese Gamelan, and Western electro-acoustic traditions exhibit no isochrony at all, but it might be argued that they do not fulfill reasonable definitions of music. For comparisons of timing in different musical cultures, see (Arom, 1991; Polak et al., 2016; Neuhoff et al., 2017). Although humans are cognitively biased toward isochrony in music (Ravignani et al., 2016a; Fitch, 2017), this bias is apparently modulated by enculturation (Jacoby and McDermott, 2017, though see Bowling et al., 2017). Finally, isochrony is often associated with motor synchronization in the literature. However, a recent medical case study has found a dissociation between perception of isochrony, among others, and audio-motor synchronization abilities (Bégel et al., 2017).

Speech is another human activity which may involve isochrony (Figure 1). The research field investigating rhythmic regularities in speech has been split for decades (Lehiste, 1977; Roach, 1982; Kotz and Schwartze, 2010, 2016; Fujii and Wan, 2014). Some scholars argue that world languages can be classified in groups exhibiting isochrony at the sentence, mora, or syllable levels (called, respectively, stress-timed, mora-timed, and syllable-timed languages, see Table 1 and Grabe and Low, 2002; Fabb and Halle, 2012). Other researchers argue the opposite, 


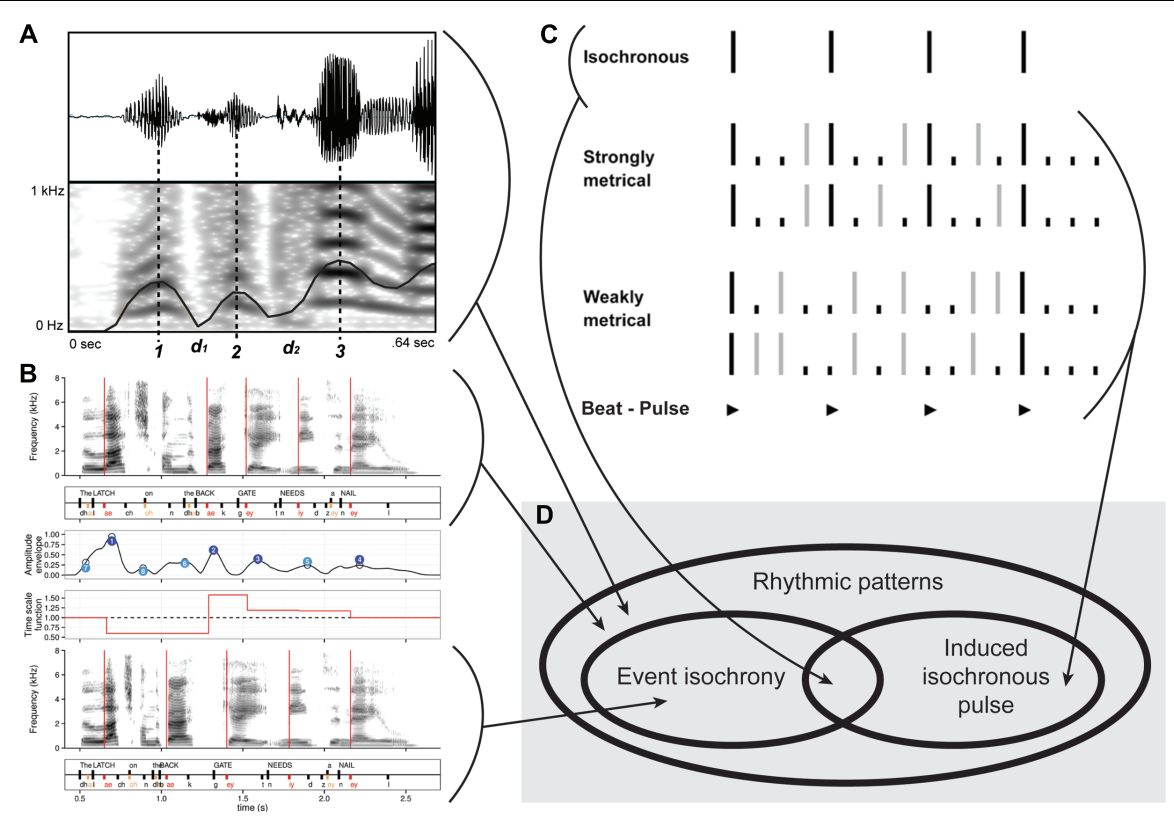

FIGURE 2 | Rhythm, isochrony and pulse. (A) Examples of a non-isochronous speech rhythmic pattern. Figure modified from Jadoul et al. (2016). (B) Examples of another non-isochronous speech rhythmic pattern (top), manipulated into isochrony (bottom). Figure modified from Aubanel et al. (2016). (C) Musical rhythm showing event isochrony (top) and non-isochronous musical pattern generating isochronous expectations (bottom). Figure modified from Launay et al. (2014). (D) Venn diagram showing the relationship among: rhythmic patterns vs. event isochrony vs. isochronous pulse perception.

namely that the speech signal is inherently anisochronous (Figures 2A,B), and the feeling of isochrony derives, for instance, from perceptual regularization rather than physical properties of the signal (Tuller and Fowler, 1980; Dauer, 1983; Jadoul et al., 2016; Brown et al., 2017). Without entering this debate here, some empirical findings are worth noticing. In particular, no matter the theoretical perspective adopted, human vocalizations can be experimentally driven toward isochrony (Jacoby and McDermott, 2017), especially when two individuals are asked to speak synchronously (Bowling et al., 2013) or perform turn-taking (Schultz et al., 2016). If speech recordings are experimentally manipulated, so that the syllable timing follows heterogeneous rhythmic patterns, isochronous speech is more intelligible than anisochronous speech (Aubanel et al., 2016). Finally, a recent experiment found evidence for isochronous timing in children's handwriting (Pagliarini et al., 2017).

A third common human activity where isochrony is hypothesized to play a role is dance (Fitch, 2016; Laland et al., 2016; Richter and Ostovar, 2016; Su, 2016a,b). Similarly to music, a series of isochronous events, such as a drum line, may provide anchor points in time used to structure dance movements (Fitch, 2016; Laland et al., 2016). Likewise, biophysical constraints on movement produce isochronous or integer ratio temporal intervals (e.g., Merker et al., 2009; Su, 2016a). This isochrony-centered perspective might however be quite specific for dance in humans inhabiting the Western world. A more inclusive approach considers dance a polyhedric behavior present in all human cultures, and whose precursors can be found in other animal species (Fink and Shackelford, 2016; Ravignani and Cook, 2016). If this approach is adopted, then isochrony might not be such an indispensable pillar of dance (Ravignani and Cook, 2016).

All the above suggests that, while isochrony might not be crucial in dance or speech, it is present in human everyday musical behavior (Figure 1). So, why is isochrony so common if it doesn't appear to serve any particular function (Fitch, 2009; Merker et al., 2009), at least in modern humans? Below we will try to analytically decompose isochrony even further in its constituent parts across disciplines.

\section{MATHEMATICS, PHYSICS, AND SIGNAL PROCESSING}

Rhythms, including isochronous ones, can be formalized mathematically (Cohen, 1962; Toussaint, 2013). From a purely information-theoretic perspective (MacKay, 2003), when producing a signal over time, isochrony minimizes the signal's entropy. A pattern of time intervals (Figure 4A) can be described by a set of interval durations and the probability of occurrence of each interval (Figure 4B). A more refined model features conditional transition probabilities (Figures 4C,D), where the duration of the upcoming interval is determined probabilistically from the duration of the previous $n$ intervals (Cohen, 1962). An example could be a specific pattern for which, given that the first and second intervals are short, there is a high probability that the third interval is long. This is not the case for isochronous sequences: no matter which interval is to be predicted, and how many past intervals are taken into account, the upcoming interval will be a constant value equal to past intervals with 


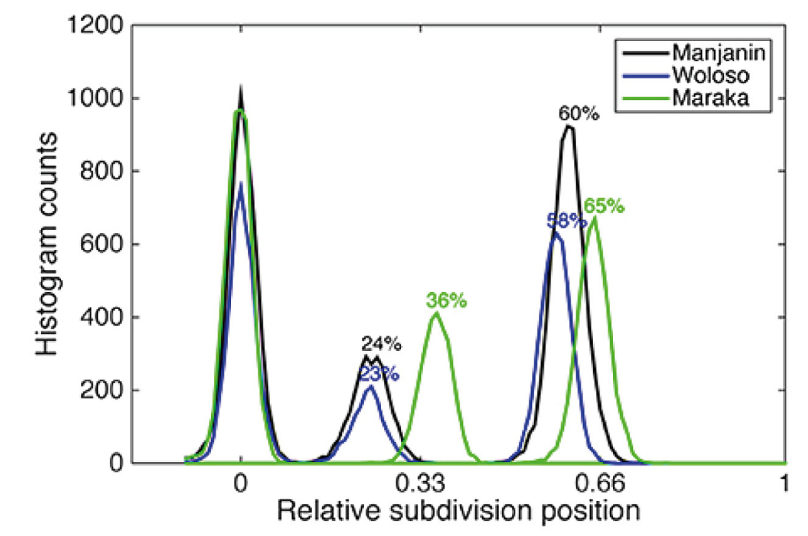

FIGURE 3 | Complex metrical structure found in djembe drumming from Mali. In this musical culture, while the top level of temporal organization is isochronous, the level below it is neither isochronous nor exhibits small integer ratios. For three musical pieces (Manjanin, Woloso, and Maraka), the figure shows the time of occurrence of other beats between two isochronous beats. Manjanin and Woloso are clearly anisochronous, while Maraka (green plot) is almost isochronous. Figure copied verbatim from Polak et al. (2016).

probability 1 . This property makes isochronous sequences, from an information-theoretic perspective, purely deterministic and predictable, granting the lowest possible entropy. In other words, conditional on a known repetition rate (i.e., tempo), isochrony minimizes entropy in rhythmic sequences.

Physics offers prime examples of isochronous processes (Strogatz, 2000). For instance, atomic clocks are based on isochronous oscillations, i.e., atomic activity regularly occurring at known frequencies (Strogatz, 2003). Events like these, reliably repeating at regular time intervals, can serve as a benchmark (Table 1): they are used by mankind to synchronize their clocks and, for our purposes, they represent the highest level of isochrony achievable by a system. In other words, empirical isochrony can be defined as synchrony, i.e., perfect co-occurrence (Ravignani, 2017a), of an empirical sequence with respect to an isochronous reference grid.

Likewise, mathematical and computational models of isochrony are quite straightforward. In the simplest case, isochrony can be mathematically generated by trigonometric functions, such as sine and cosine (Figure 5). More realistic models of isochronous human behavior involve, for instance, long-range correlations (Madison and Delignieres, 2009), and fractal scaling (Madison, 2004). Isochronous synchrony between two or more entities can be modeled using phase resetting (Sismondo, 1990; Greenfield and Roizen, 1993), period bisection (Ravignani, 2014; Ravignani et al., 2014b), coupled oscillator models (Strogatz and Stewart, 1993; Large and Kolen, 1994; Strogatz, 2000; Rouse et al., 2016; Ravignani, 2017a), and a number of other techniques (reviewed in Ravignani and Norton, 2017). In dynamical systems, isochrony ranks amongst the best understood non-linear processes. The take-home message is that isochrony, in many of its forms and variants, can be comprehensively defined by very simple mathematical expressions (and visualized geometrically, see Toussaint, 2013; Ravignani, 2017b).

\section{PHYSIOLOGY AND NEUROSCIENCE}

Human heartbeat, respiration, and locomotion all have an element of isochrony (Figure 6) in that they exhibit more regularity than random patterns but less regularity than periodic patterns in physical systems (Winfree, 1986). On the one hand, these processes can be quite regular within a short enough window of measurement (Larsson, 2012, 2014, 2015; Teie, 2016). In fact, the most commonly used measures of these physiological variables (beats, breaths or gaits per minute) assume local isochrony for the sequence analyzed. So, for example, most finger tapping studies have collected sequences of only 20-50 intervals in order to avoid the complicating drift (Wing and Kristofferson, 1973a,b; Madison, 2001). On the other hand, heartbeat, respiration and locomotion are highly dynamic and mutable in order to be functional (Figure 6); in other words, acceleration, deceleration, and phase shifts all disrupting perfect isochrony - are quite common (Strogatz, 2003).

Isochrony and synchrony are also emergent properties of the nervous system. Synchronous groups of neurons, each oscillating isochronously, are common in the brain (Buzsaki,

\section{A}

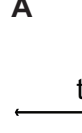

$\mathrm{P}\left(\mathrm{t}_{2} \mid \mathrm{t}_{1}\right) \mathrm{P}\left(\mathrm{t}_{3} \mid \mathrm{t}_{2}\right)$
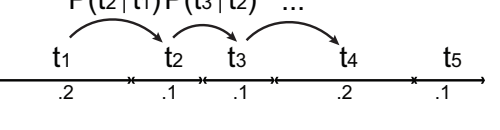

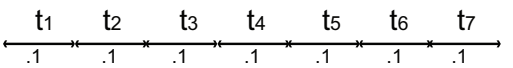

Time (s)
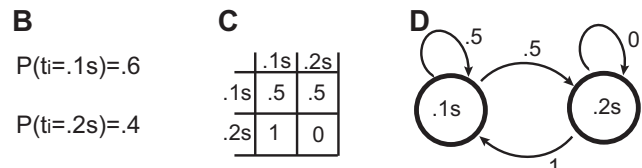

$P(\mathrm{ti}=.1 \mathrm{~s})=1$

$\mathrm{P}(\mathrm{ti}=.2 \mathrm{~s})=0$
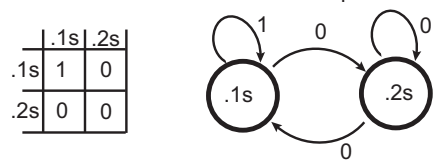

FIGURE 4 | Different ways of representing patterns of time intervals. The bottom shows an isochronous pattern, and the top a slightly more complex pattern. (A) Time series of intervals $\left(t_{1}, t_{2}, \ldots\right)$, inducing transition probabilities $P\left(t_{1}=x \mid t_{2}=y\right)$, shortened as $P\left(t_{1} \mid t_{2}\right)$. (B) Set of interval durations and probabilities of occurrence. (C) Transition matrices resulting from transition probabilities. (D) Equivalently, a probabilistic finite state machine also generates the patterns shown in (A) and described by the transition matrix in (C). 


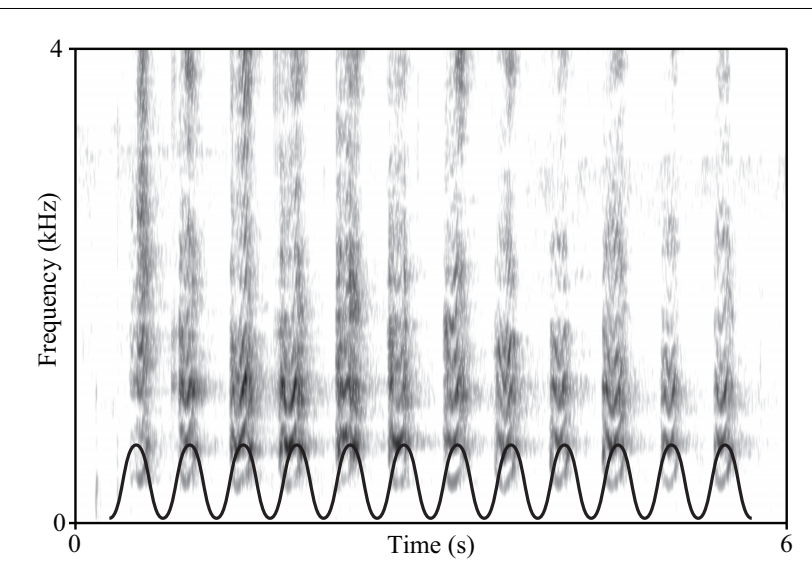

FIGURE 5 | Spectrogram of a California sea lion (Zalophus californianus) bark sequence, showing an extremely isochronous rhythm (Fourier window length $=0.02 \mathrm{~s}$ ). To highlight this metronomic regularity, we have superimposed a sinusoidal wave to the lower part of the spectrogram. The sinusoid was plotted to feature as many maxima as the number of barks: the fact that each maximum aligns with one bark provides an intuitive qualification of the isochronicity of this pinniped vocalization. (Recording collected by AR with a Zoom H6 recorder and Zoom XY-6 microphone at $44.1 \mathrm{kHZ}, 16$-bit, at Santa Cruz pier, California, on October 2016).

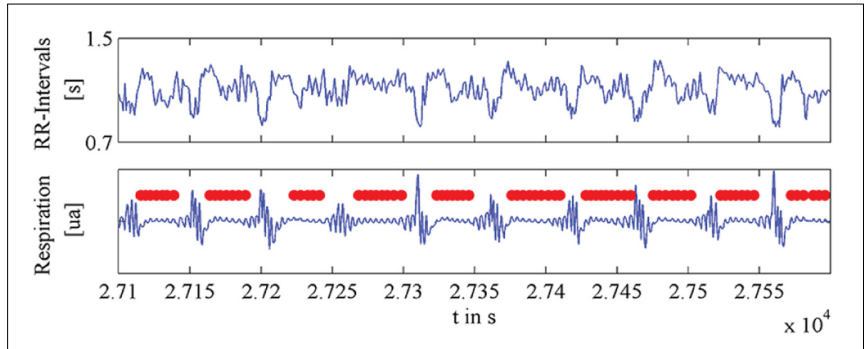

FIGURE 6 | Plot of exemplary time series of human heartbeat (top), and respiration (bottom), during and after apnea events. Figure modified from Penzel et al. (2016).

2006). At a higher level, cortico-subcortical networks are usually recruited to produce and perceive external isochronous events (Kotz and Schwartze, 2010, 2016; Nozaradan et al., 2011; Fujioka et al., 2012). Finally, some pathological states of the central nervous system are known to disrupt intentional isochrony, for instance Parkinson's disease (Grahn, 2012).

What is the neurophysiological basis for behavioral isochrony? Interesting connections between timing of vocalizations and neurophysiology have been discovered by physiologists working on non-human animals, for instance, amphibians. In some frog species, the temporal structure of courtship vocalizations is modulated by hormones (Zornik and Kelley, 2011). An outstanding question is, of course, whether these connections between temporal behavior and hormones can be found in humans, whose ethogram might be more complex than some amphibians. Recent findings in the human neurogenetics of music make this line of research quite promising (Granot et al.,
2007; Ukkola et al., 2009; Ukkola-Vuoti et al., 2013; Kanduri et al., 2015a,b).

\section{COMPARATIVE COGNITION: NON-HUMAN ANIMALS}

Some neural, behavioral and anatomical traits underlying isochronous rhythm perception and production are shared with a broad range of species (Wilson and Cook, 2016). These may either have a common evolutionary origin, or have evolved into similar traits under different evolutionary pressures (Ravignani et al., 2014b, 2016b). For instance, timing processes involving the basal ganglia and isochronous oscillations in the brain are shared with other primates and probably other animal taxa. Other traits are rare across species, only found in humans and a few other animals (Patel et al., 2009a,b). For instance, motor entrainment to an external isochronous pulse is only found sparingly in the animal kingdom (Fitch, 2015; Iversen, 2016; Ravignani et al., 2016b; Wilson and Cook, 2016).

A first, crucial difference separates human isochronous behavior from the examples of isochrony in nature provided above. This is the extent to which isochronous pattern production is driven and affected by external factors. A decaying isotope and a person walking at regular pace do not need an external oscillatory stimulus to keep producing isochronous behavior. These are cases of endogenous isochrony (Pikovsky et al., 2003), corresponding to self-sustained oscillators in physics. Conversely, the isochronous behavior exhibited by humans dancing or tapping to music is mostly exogenous: an internal pacemaker is partially corrected by externally perceived oscillatory activity (Merker et al., 2009), corresponding to forced or coupled oscillators in physics.

How about all shades of gray between these two extreme cases? Those animal species exhibiting isochrony are sparsely and heterogeneously divided among cases of endogenous and exogenous isochrony. This makes animal research key to understand the nature of human isochrony: for every particular type of isochrony found in a species, its neural mechanisms and resulting behaviors can be mapped and compared between that species and humans (Merchant et al., 2015).

Within exogenous isochrony, another distinction is between prediction and reaction (Table 1), depending on whether a timing event is produced by predicting when the next event should occur, or reacting to a previous event (Patel et al., 2009b). Humans exhibit exogenous predictive timing (Fujioka et al., 2012), while crickets exhibit exogenous reactive timing (Greenfield and Roizen, 1993). Finally, the isochronous tail wagging of dogs is quite likely to be endogenous, as external oscillatory stimuli are unlikely to affect its period or phase (cf. Buxton and Goodman, 1967; Fitch, 2009). While some species can be readily classified along the dimensions above (Rouse et al., 2016), for other species some data is available (Figure 5), though still not enough to be classified into isochrony types (e.g., Schusterman, 1977). Finally, for the majority of species, no systematic investigation of isochronous behavior has been performed. In other words, we still lack data on how most 
species produce and perceive isochrony under a wide range of different conditions, which would be diagnostic to the underlying mechanisms and limitations.

In animal research, isochrony has been investigated using two main methods: observing natural behavior and training animals to produce specific temporal sequences. Isochrony as natural behavior in other animal species has long been studied, though its relevance to human rhythm has been pointed out only recently (Madison, 2004; Merker et al., 2009; Ravignani et al., 2014b). In fact, many animals signal over time in a precisely isochronous fashion (e.g., see Figure 5). 'Isochronous species' span crickets, frogs, fireflies, birds, crabs, and marine mammals (Schusterman, 1977; Sismondo, 1990; Greenfield and Roizen, 1993; Strogatz, 2003; Merker et al., 2009; Kahn et al., 2014; Norton and Scharff, 2016). As these studies are often purely behavioral and observational, rarely targeting neurobiological brain mechanisms, it is difficult to know whether isochrony is endogenous, exogenous, predictive or reactive.

An alternative is to test animals' capacities to produce isochrony in a controlled experimental setup. This is often done in conjunction with synchronization experiments. The animals are trained to produce specific isochronous behaviors, often with the purpose of entraining to a musical beat, and are then tested in their ability to generalize to different tempi and levels of jitter (Wilson and Cook, 2016). The only irrefutable results of exogenous predictive isochrony in any animal species are three: humans, a sea lion and a cockatoo (Patel et al., 2009a; Cook et al., 2013). Trained isochrony due instead to reactive timing might be more common: several species appear capable of producing series of temporal intervals of equal duration (Hasegawa et al., 2011; Hattori et al., 2015).

\section{ISOCHRONY IN INTERACTION}

In human communication, two opposing functions affect the structure of the signal: expressivity and compressibility (Table $\mathbf{1}$ and Kirby et al., 2015). Expressivity influences the amount of information content, hence semantics, which ideally should be maximized (Kirby et al., 2015). Compressibility refers to the density of information transmitted: intuitively, it is cost efficient to transmit the same amount of information in its shortest or maximally compressed form (MacKay, 2003). In other words, signalers would ideally broadcast the maximum quantity of information, using the least possible amount of signal. This tradeoff is further modulated by redundancy: a maximally compressible communication system with no redundancy can be irreversibly corrupted by a minimal transmission error. Hence, a signaler might not want to completely minimize entropy in order to leave room for redundancy.

To what extent can compressibility, expressivity, and redundancy account for human isochrony (Figure 7)? Mathematically, isochronous signals maximize redundancy and minimize entropy, but leave almost no room for expressivity. Comparatively, when human participants develop signaling systems in communication experiments, no expressivity leads to maximum compressibility (Kirby et al., 2008, 2015). This, by analogy, would dismiss isochronous pattern production as an expressive communication system (Figure 7), i.e., a system where signals are mapped to meanings (but see Bharucha and Pryor, 1986; Horr and Di Luca, 2015). However, the meaning of the transmitted message could lie in the signal emission per se, rather than that being broadcasted through the signal. This is the concept behind 'signaling signalhood' (Scott-Phillips et al., 2009): the message of an isochronous pattern is its 'isochronicity', instead of being used in referential communication. Consider a hypothetical example. Take rhythmic sequences composed of only two durational intervals (such as the first two intervals of a sequence in Figure 4A). The set of all two sequences could be used for communicative purposes in two main ways. In the first, more common case of 'referential communication', the duration of the two intervals could encode different conceptual properties. For instance, the first interval could be used to encode the size of a referent, while the second its brightness. Hence, a rhythm composed of a short and a long interval would communicate a small, dark object, while a long and a short interval would refer to a large bright object. This variability in the lengths of the intervals would grant expressivity. However, if isochronous sequences were the signals most frequently transmitted, this way of encoding signals could not be expressive, because all objects would end up being encoded as having an average size and brightness. In contrast, in the second case of 'signaling signalhood, the two intervals would be used to communicate precision in isochronous pattern production, i.e., to signal isochronicity. Hence, a pattern as the bottom of Figure $4 \mathbf{A}$ would signal high precision in isochrony, while the top pattern in Figure 4A would signal poor isochrony. From this point of view, human perception and production of isochronous patterns might better fit the second, signaling signalhood framework, rather than the first, referential communication framework.

There is a close match between the most precise levels of isochrony that humans are capable of producing and those they are capable of perceiving (Madison and Merker, 2002; Merker et al., 2009). This match also offers some support for the hypothesis that isochrony might have been shaped for communicative purposes. In other words, a communication system, and in particular one that takes advantage of, and evolves from, perceptual biases (Ryan, 1998), will show a match between features of the signal and the capacities to perceive those features. For example, the plumages of many bird species reflect ultraviolet light, which humans and other species cannot see, while conspecific birds can readily perceive and use to select a mate (Andersson and Amundsen, 1997; Vorobyev et al., 1998; Eaton, 2005). We hypothesize that an analogous process might have resulted from isochrony (expanding on Merker, 1999, 2000), if this were a communicative trait. In particular, a communication system employed to transmit information about deviations from an isochronous pulse would evolve toward levels of precision comparable between production and perception (Merker, 1999, 2000). This comparable precision is exactly what can be observed in human motoric and perceptual isochrony (Madison and Merker, 2002; Merker et al., 2009), offering some preliminary, indirect support for a possible communicative function of isochrony. 


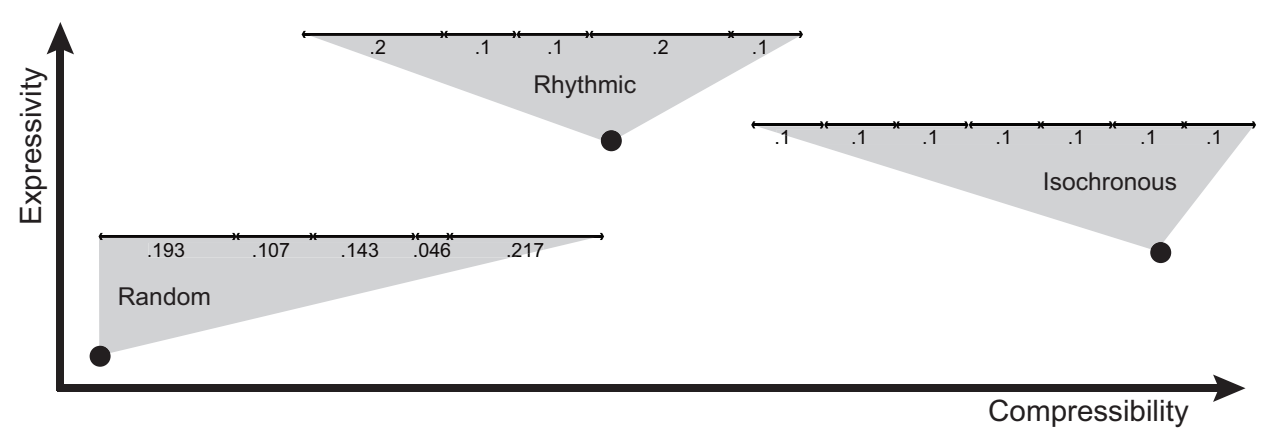

FIGURE 7 | Compressibility-expressivity tradeoff for temporal patterns. Three examples of timing patterns are provided, placing them in this compressibility-expressivity space. For each example, the black dot denotes the approximate location of the pattern. Each horizontal line represents one pattern, with the duration of each chunk expressed in seconds below it. Patterns used for communicative purposes are predicted to lie on or near the main diagonal, and patterns in the top-left and bottom-right quadrants of the diagram are hence not predicted to serve communicative purposes.

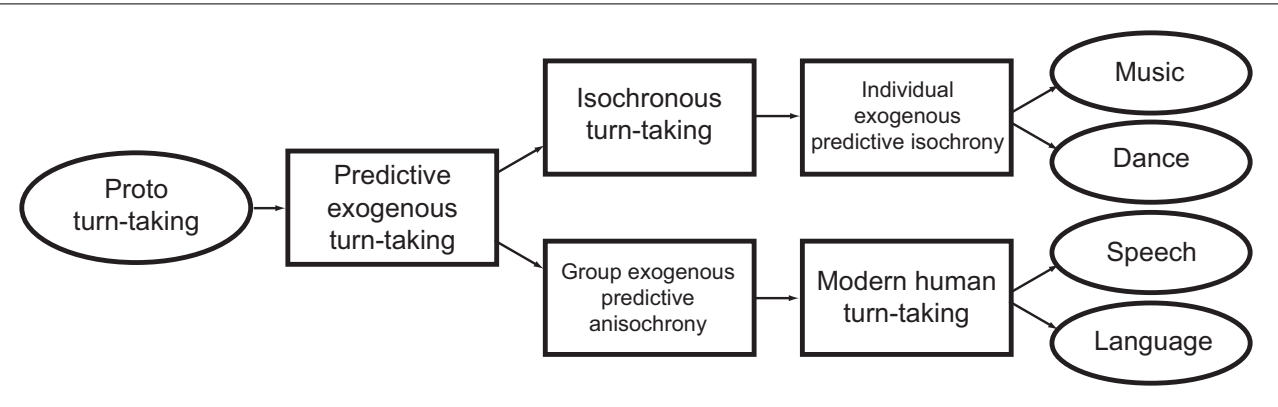

FIGURE 8 | Flux diagram of hypothetical precursors to isochronous synchrony and turn-taking. A hypothetical form of 'proto turn-taking' branches in two, leading to isochrony, as found in music and dance, and anisochronous turn-taking, as found in language and speech.

Isochrony does not appear to be used in the overt communication of modern humans, but might have played a role in some form of communication employed by our ancestors. In fact, isochrony is the optimal way to establish synchronized group signaling because it makes the duration of next interval perfectly predictable by another person or conspecific (Merker et al., 2009). This musical perspective on the evolution of isochrony connects to turn-taking, which is a crucial component of human language (Figure 8). Turn-taking allows speakers to effectively interact in conversation: it avoids that speakers' utterances overlap, while still enabling utterances to occur within a reasonable amount of time from each other. Interestingly, turntaking in language is both predictive and exogenous, but seems to lack isochrony, except maybe in a few special cases. Still, turntaking exhibits a particular temporal structure (Stivers et al., 2009; Levinson and Torreira, 2015). This structure appears to arise by a constant $200 \mathrm{~ms}$ lag (Figure 9C) between the ends and starts of utterances across cultures (Stivers et al., 2009), rather than a lag between the starts of consecutive utterances. This fixed-interval delay contrasts with the slightly positive or negative lags found in animal synchronization experiments (Figures 9A,B), and the anticipatory reaction in human musical synchronization. So, in modern humans, turn-taking is far from isochrony (except for when it is a by product of utterances having the same duration within and between speakers), but it might promote isochrony (Schultz et al., 2016). This makes turn-taking in modern organisms a potential approach to understand the evolution of isochrony (see Figure 8).

\section{EVOLUTIONARY HYPOTHESES AND FUTURE EMPIRICAL WORK}

In conclusion, isochrony does not appear to relate to any current selection pressure. This is not surprising: a large number of evolved, heritable traits do not readily map to clear selection pressures in extant species. Instead, the pressures giving rise to isochrony might be sought in ancient humans, operating at some point between now and the split between our ancestor and that of chimpanzees/bonobos. The most articulate hypothesis to date proposes a multistage model (Merker, 1999, 2000; Merker et al., 2009). According to this, a recent ancestor to modern Homo sapiens would have been exposed to a selection pressure to attract migrating conspecific females. Accordingly, group vocalizations would have provided a conspicuously loud signal. The more individuals that managed to synchronize their calls, the greater the sound intensity, and the farther its reach in the terrain. In turn, the easiest way to achieve synchronization is to produce an isochronous signal, which is maximally predictable by the other callers, leading to an 

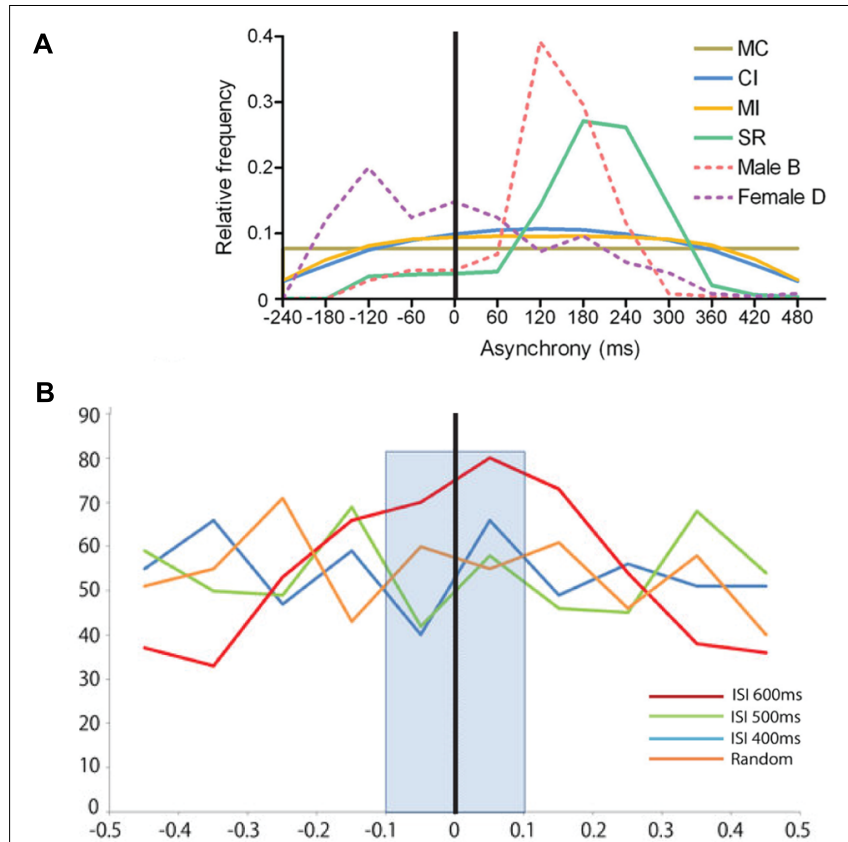

C

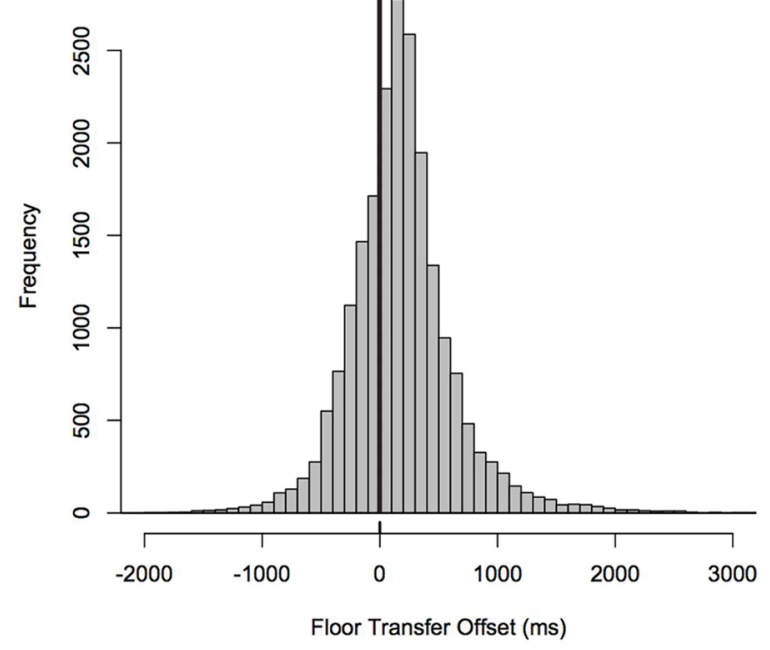

FIGURE 9 | Onset asynchronies in (A) a synchronization experiment in budgerigars (each bird, Male B and Female D, is a dashed line), (B) a tapping experiment in one individual chimpanzee, and (C) turn-taking in human conversation. Figure panels modified from Hasegawa et al. (2011) for (A), Hattori et al. (2013) for (B), and Levinson and Torreira (2015) for (C).

isochronous, synchronous chorus (Merker et al., 2009). We further extend this idea, suggesting that early stages of vocal coordination could be precursors to two modern human traits: isochronous signaling in music and anisochronous turn-taking in language (Figure 8).

Our perspective focuses on the function of isochrony (Merker et al., 2009; Ravignani et al., 2014b), rather than its underlying ontogeny and phylogeny (Tinbergen, 1963). Hence, most ideas presented here are in principle compatible with other hypotheses focused on developmental trajectories or mechanisms
(Iversen, 2016; Teie, 2016). The functional reason why isochrony appeared in human evolution might therefore not be directly derivable from how isochrony appears in development, or from its neurobehavioral mechanisms. The mechanisms underlying isochrony which manifest through development (Eerola et al., 2006) might also be reflected in evolution, though they do not need to be (see Gould, 1977, and subsequent debate).

Research on modern humans provides some grounds, in principle, for isochrony to have been an evolutionary selected trait. In particular, isochronous timing seems quite variable across individuals, and enhanced by learning (Max and Yudman, 2003; Manning and Schutz, 2016; Tierney et al., 2017). Individual differences and learning plasticity are neither necessary nor sufficient conditions to show that a trait, such as isochrony, underwent evolution by natural selection. However, individual differences are often a prerequisite for natural selection to act on a trait. Likewise, learning plasticity can be an outcome of an evolutionary process acting on behavior and cognition, rather than on a physical trait.

For the purpose of the present paper, it would be interesting to find the genetic and neuro-hormonal biological substrates responsible for perception and production of isochronous behavior both in humans and other animals. In fact, as isochrony appears as a relatively simple behavioral trait, study of its neurogenetic and hormonal substrates might prove an initial building block to understand rhythm more in general.

Our suggestions can be readily tested along several strands of empirical research in humans. Temporal interactions in groups of animals are known to lead to isochrony as one of the equilibrium outcomes (Sismondo, 1990; Greenfield and Roizen, 1993; Kahn et al., 2014). Human data on turn-taking may be re-analyzed, asking whether the isochrony of each partner entails the best predictability of turn-taking vs. constant-lag alternation. Likewise, the large body of research on isochrony perception and production across modalities and domains may be synthetized (Iversen et al., 2015; Celma-Miralles et al., 2016), to examine (1) the limits and boundaries of the human sense of isochrony, and (2) which experiments are lacking that would entail comparability across domains and modalities. In general, the field of rhythm would benefit by a tighter connection between individual and group processes: individual behavioral traits do not evolve in a vacuum, and individual timing might be modulated by social factors (Ravignani et al., 2014a,b; Ravignani and Cook, 2016; Schirmer et al., 2016). For instance, in some primate and avian species, singing is accurately timed with the group depending on the sex and social status of each individual singer (Mann et al., 2006; Gamba et al., 2016). With this logic in mind, we can list a number of specific outstanding questions:

- Do individual and group isochrony influence each other (Ravignani et al., 2014b)? How are they related at a mechanistic, functional, developmental and phylogenetic level (Kirschner and Tomasello, 2009; Ravignani et al., 2016b)?

- What are the effects of training, conformity, and social cues on the perception and production of isochronous behaviors (Schirmer et al., 2016)? 
- How do different individual group interaction modes, for instance coordination and competition, map on to temporal patterns produced, such as isochrony (Ravignani et al., 2014a)?

These questions are not only relevant from the perspective of human cognitive neuroscience and animal behavior. They are also key to test evolutionary hypotheses, where the fitness landscape might be influenced by social factors and cultural niches (Tomasello, 2009; Boyd et al., 2011; Kendal, 2011).

Finally, while selection pressures in our ancestors are difficult to reconstruct, their effects might still be observable in the behavioral tendencies, genome and neuroendocrine system of modern humans (Holmquist and Vestin, 2010; Madison, 2011; Björk, 2013; Madison et al., 2017, in press). For instance, recent studies have mapped musical and rhythmic phenotypes to genes and hormonal profiles (Mosing et al., 2015; Miani, 2016a,b); more focused studies linking biology and psychology are needed for the specific trait(s) underlying isochrony.

\section{REFERENCES}

Andersson, S., and Amundsen, T. (1997). Ultraviolet colour vision and ornamentation in bluethroats. Proc. R. Soc. Lond. B Biol. Sci. 264, 1587-1591. doi: $10.1098 / \mathrm{rspb} .1997 .0221$

Arnal, L. H., and Giraud, A.-L. (2012). Cortical oscillations and sensory predictions. Trends Cogn. Sci. 16, 390-398. doi: 10.1016/j.tics.2012. 05.003

Arom, S. (1991). African Polyphony and Polyrhythm: Musical Structure and Methodology, Trans. M. Thom, B. Tuckett, and R. Boyd (Cambridge: Cambridge university press). doi: 10.1017/CBO9780511518317

Aubanel, V., Davis, C., and Kim, J. (2016). Exploring the role of brain oscillations in speech perception in noise: intelligibility of isochronously retimed speech. Front. Hum. Neurosci. 10:430. doi: 10.3389/fnhum.2016.00430

Bégel, V., Benoit, C.-E., Correa, A., Cutanda, D., Kotz, S. A., and Dalla Bella, S. (2017). "Lost in time" but still moving to the beat. Neuropsychologia 94, 129-138. doi: 10.1016/j.neuropsychologia.2016.11.022

Bharucha, J. J., and Pryor, J. H. (1986). Disrupting the isochrony underlying rhythm: an asymmetry in discrimination. Percept. Psychophys. 40, 137-141. doi: 10.3758/BF03203008

Björk, J. (2013). Could Musical Mastery Affect how Attractive A Person is Rated As A Prospective Partner?. B.Sc. thesis, Umeå University, Umeå.

Bolton, T. L. (1894). Rhythm. Am. J. Psychol. 6, 145-238. doi: 10.2307/1410948

Bowling, D. L., Herbst, C. T., and Fitch, W. T. (2013). Social origins of rhythm? synchrony and temporal regularity in human vocalization. PLOS ONE 8:e80402. doi: 10.1371/journal.pone.0080402

Bowling, D. L., Hoeschele, M., Gill, K. Z., and Fitch, W. T. (2017). The nature and nurture of musical consonance. Music Percept. 35, 118-121. doi: 10.1525/mp. 2017.35.1.118

Boyd, R., Richerson, P. J., and Henrich, J. (2011). The cultural niche: why social learning is essential for human adaptation. Proc. Natl. Acad. Sci. U.S.A. 108(Suppl. 2), 10918-10925. doi: 10.1073/pnas.11002 90108

Brown, S., Pfordresher, P. Q., and Chow, I. (2017). A musical model of speech rhythm. Psychomusicology 27, 95. doi: 10.1037/pmu0000175

Buxton, D. F., and Goodman, D. C. (1967). Motor function and the corticospinal tracts in the dog and raccoon. J. Comp. Neurol. 129, 341-360. doi: 10.1002/cne. 901290405

Buzsaki, G. (2006). Rhythms of the Brain. Oxford: Oxford University Press. doi: 10.1093/acprof:oso/9780195301069.001.0001

\section{AUTHOR CONTRIBUTIONS}

AR wrote a first draft of the manuscript. GM provided references, guidance, and advice, and edited the manuscript.

\section{FUNDING}

AR has received funding from the European Union's Horizon 2020 research and innovation program under the Marie Skłodowska-Curie grant agreement No. 665501 with the research Foundation Flanders (FWO) (Pegasus ${ }^{2}$ Marie Curie fellowship $12 \mathrm{~N} 5517 \mathrm{~N}$ awarded to AR), a visiting fellowship in Language Evolution from the Max Planck Society (awarded to AR), and ERC grant 283435 ABACUS (awarded to Bart de Boer).

\section{ACKNOWLEDGMENT}

AR is grateful to Steve Levinson for advice and support, and to Bill Thompson and Heikki Rasilo for the stimulating discussions in Tennessee, which inspired this paper.

Celma-Miralles, A., de Menezes, R. F., and Toro, J. M. (2016). Look at the beat, feel the meter: top-down effects of meter induction on auditory and visual modalities. Front. Hum. Neurosci. 10:108. doi: 10.3389/fnhum.2016. 00108

Cohen, J. E. (1962). Information theory and music. Behav. Sci. 7, 137-163. doi: 10.1002/bs.3830070202

Cook, P., Rouse, A., Wilson, M., and Reichmuth, C. J. (2013). A California sea lion (Zalophus californianus) can keep the beat: motor entrainment to rhythmic auditory stimuli in a non vocal mimic. J. Comp. Psychol. 127, 1-16. doi: 10.1037/ a0032345

Dauer, R. M. (1983). Stress-timing and syllable-timing reanalyzed. J. Phonet. 11, 51-62.

Eaton, M. D. (2005). Human vision fails to distinguish widespread sexual dichromatism among sexually "monochromatic" birds. Proc. Natl. Acad. Sci. U.S.A. 102, 10942-10946. doi: 10.1073/pnas.0501891102

Eerola, T., Luck, G., and Toiviainen, P. (2006). An investigation of pre-schoolers' corporeal synchronization with music. Paper Presented at the Proceedings of the 9th International Conference on Music Perception and Cognition, Bologna.

Fabb, N., and Halle, M. (2012). "Grouping in the stressing of words, in metrical verse, and in music," in Language and Music as Cognitive Systems, eds P. Rebuschat, M. Rohmeier, J. A. Hawkins, and I. Cross (Oxford: Oxford University Press), 4-21.

Fink, B., and Shackelford, T. K. (2016). Why did dance evolve? a comment on Laland, Wilkins, and Clayton (2016). Evol. Psychol. Sci. 3, 147-148. doi: 10.1007/ s40806-016-0075-3

Fitch, W. T. (2009). The Biology and Evolution of Rhythm: Unraveling a Paradox Language and Music as Cognitive Systems. Oxford: Oxford University Press.

Fitch, W. T. (2015). "The biology and evolution of musical rhythm: an update," in Structures in the Mind: Essays on Language, Music, and Cognition in Honor of Ray Jackendoff, eds D. I. Toivonen, P. Csúri, and E. van der Zee (Cambridge, MA: MIT Press), 293-324.

Fitch, W. T. (2016). Dance, music, meter and groove: a forgotten partnership. Front. Hum. Neurosci. 10:64. doi: 10.3389/fnhum.2016.00064

Fitch, W. T. (2017). Cultural evolution: lab-cultured musical universals. Nat. Hum. Behav. 1, 1-2. doi: 10.1038/s41562-016-0018

Friberg, A., and Sundberg, J. (1995). Time discrimination in a monotonic, isochronous sequence. J. Acoust. Soc. Am. 98, 2524-2531. doi: 10.1121/1.413218

Fujii, S., and Wan, C. Y. (2014). The role of rhythm in speech and language rehabilitation: the SEP hypothesis. Front. Hum. Neurosci. 8:777. doi: 10.3389/ fnhum.2014.00777 
Fujioka, T., Trainor, L. J., Large, E. W., and Ross, B. (2012). Internalized timing of isochronous sounds is represented in neuromagnetic beta oscillations. J. Neurosci. 32, 1791-1802. doi: 10.1523/JNEUROSCI.4107-11.2012

Gamba, M., Torti, V., Estienne, V., Randrianarison, R. M., Valente, D., Rovara, P., et al. (2016). The indris have got rhythm! Timing and pitch variation of a primate song examined between sexes and age classes. Front. Neurosci. 10:249. doi: 10.3389/fnins.2016.00249

Gould, S. J. (1977). Ontogeny and Phylogeny. Cambridge, MA: Harvard University Press.

Grabe, E., and Low, E. L. (2002). "Durational variability in speech and the rhythm class hypothesis," in Papers in Laboratory Phonology, 7, eds N. Warner and C. Gussenhoven (Berlin: Mouton de Gruyter), 515-546.

Grahn, J. A. (2012). Neural mechanisms of rhythm perception: current findings and future perspectives. Top. Cogn. Sci. 4, 585-606. doi: 10.1111/j.1756-8765. 2012.01213.x

Granot, R. Y., Frankel, Y., Gritsenko, V., Lerer, E., Gritsenko, I., BachnerMelman, R., et al. (2007). Provisional evidence that the arginine vasopressin 1a receptor gene is associated with musical memory. Evol. Hum. Behav. 28, 313-318. doi: 10.1016/j.evolhumbehav.2007.05.003

Greenfield, M. D., and Roizen, I. (1993). Katydid synchronous chorusing is an evolutionarily stable outcome of female choice. Nature 364, 618-620. doi: $10.1038 / 364618 \mathrm{a} 0$

Hasegawa, A., Okanoya, K., Hasegawa, T., and Seki, Y. (2011). Rhythmic synchronization tapping to an audio-visual metronome in budgerigars. Sci. Rep. 1:120. doi: 10.1038/srep00120

Hattori, Y., Tomonaga, M., and Matsuzawa, T. (2013). Spontaneous synchronized tapping to an auditory rhythm in a chimpanzee. Sci. Rep. 3:1566. doi: 10.1038/ srep01566

Hattori, Y., Tomonaga, M., and Matsuzawa, T. (2015). Distractor effect of auditory rhythms on self-paced tapping in chimpanzees and humans. PLOS ONE 10:e0130682. doi: 10.1371/journal.pone.0130682

Holmquist, J., and Vestin, M. (2010). Will Musical Skill Attract Mates? An Experimental Study of Mate Preferences as a Function of Musical Ability. M.Sc. thesis, Umeå University, Umeå.

Honing, H., Ladinig, O., Háden, G. P., and Winkler, I. (2009). Is beat induction innate or learned? Ann. N. Y. Acad. Sci. 1169, 93-96. doi: 10.1111/j.1749-6632. 2009.04761.x

Horr, N. K., and Di Luca, M. (2015). Taking a long look at isochrony: perceived duration increases with temporal, but not stimulus regularity. Atten. Percept. Psychophys. 77, 592-602. doi: 10.3758/s13414-014-0787-z

Iversen, J. R. (2016). "In the beginning was the beat: evolutionary origins of musical rhythm in humans," in The Cambridge Companion to Percussion, ed. R. Hartenberger (Cambridge: Cambridge University Press).

Iversen, J. R., Patel, A. D., Nicodemus, B., and Emmorey, K. (2015). Synchronization to auditory and visual rhythms in hearing and deaf individuals. Cognition 134, 232-244. doi: 10.1016/j.cognition.2014.10.018

Jacoby, N., and McDermott, J. H. (2017). Integer ratio priors on musical rhythm revealed cross-culturally by iterated reproduction. Curr. Biol. 27, 359-370. doi: 10.1016/j.cub.2016.12.031

Jadoul, Y., Ravignani, A., Thompson, B., Filippi, P., and de Boer, B. (2016). Seeking temporal predictability in speech: comparing statistical approaches on 18 world languages. Front. Hum. Neurosci. 10:586. doi: 10.3389/fnhum.2016.00586

Kahn, A. T., Holman, L., and Backwell, P. R. (2014). Female preferences for timing in a fiddler crab with synchronous courtship waving displays. Anim. Behav. 98, 35-39. doi: 10.1016/j.anbehav.2014.09.028

Kanduri, C., Kuusi, T., Ahvenainen, M., Philips, A. K., Lähdesmäki, H., and Järvelä, I. (2015a). The effect of music performance on the transcriptome of professional musicians. Sci. Rep. 5:9506. doi: 10.1038/srep09506

Kanduri, C., Raijas, P., Ahvenainen, M., Philips, A. K., Ukkola-Vuoti, L., Lähdesmäki, H., et al. (2015b). The effect of listening to music on human transcriptome. PeerJ 3:e830. doi: 10.7717/peerj.830

Kendal, J. R. (2011). Cultural niche construction and human learning environments: Investigating sociocultural perspectives. Biol. Theory 6, 241-250. doi: 10.1007/s13752-012-0038-2

Kirby, S., Cornish, H., and Smith, K. (2008). Cumulative cultural evolution in the laboratory: an experimental approach to the origins of structure in human language. Proc. Natl. Acad. Sci. U.S.A. 105, 10681-10686. doi: 10.1073/pnas. 0707835105
Kirby, S., Tamariz, M., Cornish, H., and Smith, K. (2015). Compression and communication in the cultural evolution of linguistic structure. Cognition 141, 87-102. doi: 10.1016/j.cognition.2015.03.016

Kirschner, S., and Tomasello, M. (2009). Joint drumming: social context facilitates synchronization in preschool children. J. Exp. Child Psychol. 102, 299-314. doi: 10.1016/j.jecp.2008.07.005

Kotz, S. A., and Schwartze, M. (2010). Cortical speech processing unplugged: a timely subcortico-cortical framework. Trends Cogn. Sci. 14, 392-399. doi: 10.1016/j.tics.2010.06.005

Kotz, S. A., and Schwartze, M. (2016). "Motor-timing and sequencing in speech production: a general-purpose framework," in Neurobiology of Language, eds G. Hickok and S. L. Small (San Diego, CA: Academic Press), 717-724. doi: 10.1016/B978-0-12-407794-2.00057-2

Laland, K., Wilkins, C., and Clayton, N. (2016). The evolution of dance. Curr. Biol. 26, R5-R9. doi: 10.1016/j.cub.2015.11.031

Large, E. W., and Kolen, J. F. (1994). Resonance and the perception of musical meter. Conn. Sci. 6, 177-208. doi: 10.1080/09540099408915723

Larsson, M. (2012). Incidental sounds of locomotion in animal cognition. Anim. Cogn. 15, 1-13. doi: 10.1007/s10071-011-0433-2

Larsson, M. (2014). Self-generated sounds of locomotion and ventilation and the evolution of human rhythmic abilities. Anim. Cogn. 17, 1-14. doi: 10.1007/ s10071-013-0678-z

Larsson, M. (2015). Tool-use-associated sound in the evolution of language. Anim. Cogn. 18, 993-1005. doi: 10.1007/s10071-015-0885-x

Launay, J., Grube, M., and Stewart, L. (2014). Dysrhythmia: a specific congenital rhythm perception deficit. Front. Psychol. 5:18. doi: 10.3389/fpsyg.2014.00018

Lehiste, I. (1977). Isochrony reconsidered. J. Phonet. 5, 253-263.

Levinson, S. C., and Torreira, F. (2015). Timing in turn-taking and its implications for processing models of language. Front. Psychol. 6:731. doi: 10.3389/fpsyg. 2015.00731

MacKay, D. J. (2003). Information Theory, Inference and Learning Algorithms. Cambridge: Cambridge University Press.

Madison, G. (2001). Variability in isochronous tapping: higher order dependencies as a function of intertap interval. J. Exp. Psychol. 27, 411-422. doi: 10.1037/ 0096-1523.27.2.411

Madison, G. (2004). Fractal modeling of human isochronous serial interval production. Biol. Cybern. 90, 105-112. doi: 10.1007/s00422-003-0453-3

Madison, G. (2009). An auditory illusion of infinite tempo change based on multiple temporal levels. PLOS ONE 4:e8151. doi: 10.1371/journal.pone. 0008151

Madison, G. (2011). "Cause and affect: a functional perspective on music and emotion," in Art and the Senses, eds F. Bacci and D. Melcher (Oxford: Oxford University Press), 329-350.

Madison, G. (2014). Sensori-motor synchronisation variability decreases as the number of metrical levels in the stimulus signal increases. Acta Psychol. 147, 10-16. doi: 10.1016/j.actpsy.2013.10.002

Madison, G., and Delignieres, D. (2009). Auditory feedback affects the long-range correlation of isochronous serial interval production: support for a closed-loop or memory model of timing. Exp. Brain Res. 193, 519-527. doi: 10.1007/s00221008-1652-x

Madison, G., Holmquist, J., and Vestin, M. (in press). Musical improvisation skill in a prospective partner is associated with mate value and preferences, consistent with sexual selection and parental investment theory: implications for the origin of music. Evol. Hum. Behav.

Madison, G., and Merker, B. (2002). On the limits of anisochrony in pulse attribution. Psychol. Res. 66, 201-207. doi: 10.1007/s00426-001-0085-y

Madison, G., and Merker, B. (2004). Human sensorimotor tracking of continuous subliminal deviations from isochrony. Neurosci. Lett. 370, 69-73. doi: 10.1016/ j.neulet.2004.07.094

Madison, G., Ullén, F., and Merker, B. (2017). "Metrically structured time and entrainment," in The Routledge Companion to Embodied Music Interaction, eds M. Lesaffre, M. Leman, and P. Maes (London: Routledge).

Mann, N. I., Dingess, K. A., and Slater, P. (2006). Antiphonal four-part synchronized chorusing in a Neotropical wren. Biol. Lett. 2, 1-4. doi: 10.1098/ rsbl.2005.0373

Manning, F. C., and Schutz, M. (2016). Trained to keep a beat: movement-related enhancements to timing perception in percussionists and non-percussionists. Psychol. Res. 80, 532-542. doi: 10.1007/s00426-015-0678-5 
Max, L., and Yudman, E. M. (2003). Accuracy and variability of isochronous rhythmic timing across motor systems in stuttering versus nonstuttering individuals. J. Speech Lang. Hear. Res. 46, 146-163. doi: 10.1044/10924388(2003/012)

McAuley, J. D. (2010). Tempo and Rhythm Music Perception. Berlin: Springer, 165-199. doi: 10.1007/978-1-4419-6114-3_6

Merchant, H., Grahn, J., Trainor, L., Rohrmeier, M., and Fitch, W. T. (2015). Finding the beat: a neural perspective across humans and non-human primates. Philos. Trans. R. Soc. B 370:20140093. doi: 10.1098/rstb.2014.0093

Merker, B. (1999). Synchronous chorusing and the origins of music. Music. Sci. 3, 59-73. doi: 10.1177/10298649000030S105

Merker, B. (2000). "Synchronous chorusing and human origins," in The Origins of Music, eds N. Wallin, B. Merker, and S. Brown (Cambridge, MA: MIT Press), $315-327$.

Merker, B. (2014). Groove or swing as distributed rhythmic consonance: introducing the groove matrix. Front. Hum. Neurosci. 8:454. doi: 10.3389/ fnhum.2014.00454

Merker, B., Madison, G., and Eckerdal, P. (2009). On the role and origin of isochrony in human rhythmic entrainment. Cortex 45, 4-17. doi: 10.1016/j. cortex.2008.06.011

Miani, A. (2016a). The Impact of Sex and Menstrual Cycle on Rhythmic Synchronization: An Experimental Approach. Master thesis, Aarhus University, Aarhus.

Miani, A. (2016b). Sexual arousal and rhythmic synchronization: a possible effect of vasopressin. Med. Hypotheses 93, 122-125. doi: 10.1016/j.mehy.2016.05.030

Mosing, M. A., Verweij, K. J., Madison, G., Pedersen, N. L., Zietsch, B. P., and Ullén, F. (2015). Did sexual selection shape human music? Testing predictions from the sexual selection hypothesis of music evolution using a large genetically informative sample of over 10,000 twins. Evol. Hum. Behav. 36, 359-366. doi: 10.1016/j.evolhumbehav.2015.02.004

Motz, B. A., Erickson, M. A., and Hetrick, W. P. (2013). To the beat of your own drum: cortical regularization of non-integer ratio rhythms toward metrical patterns. Brain Cogn. 81, 329-336. doi: 10.1016/j.bandc.2013.01.005

Neuhoff, H., Polak, R., and Fischinger, T. (2017). Perception and evaluation of timing patterns in drum ensemble music from mali. Music Percept. 34, 438-451. doi: $10.1525 / \mathrm{mp} .2017 .34 .4 .438$

Norton, P., and Scharff, C. (2016). "Bird song metronomics": isochronous organization of zebra finch song rhythm. Front. Neurosci. 10:309. doi: 10.3389/ fnins.2016.00309

Nozaradan, S., Peretz, I., Missal, M., and Mouraux, A. (2011). Tagging the neuronal entrainment to beat and meter. J. Neurosci. 31, 10234-10240. doi: 10.1523/ JNEUROSCI.0411-11.2011

Pagliarini, E., Scocchia, L., Vernice, M., Zoppello, M., Balottin, U., Bouamama, S., et al. (2017). Children's first handwriting productions show a rhythmic structure. Sci. Rep. 7:5516. doi: 10.1038/s41598-017-05105-6

Patel, A. D., Iversen, J. R., Bregman, M. R., and Schulz, I. (2009a). Experimental evidence for synchronization to a musical beat in a nonhuman animal. Curr. Biol. 19, 827-830. doi: 10.1016/j.cub.2009.03.038

Patel, A. D., Iversen, J. R., Bregman, M. R., and Schulz, I. (2009b). Studying synchronization to a musical beat in nonhuman animals. Ann. N. Y. Acad. Sci. 1169, 459-469. doi: 10.1111/j.1749-6632.2009.04581.x

Penzel, T., Kantelhardt, J. W., Bartsch, R. P., Riedl, M., Kraemer, J. F., Wessel, N., et al. (2016). Modulations of heart rate, ECG, and cardio-respiratory coupling observed in polysomnography. Front. Physiol. 7:460. doi: 10.3389/fphys.2016. 00460

Pikovsky, A., Rosenblum, M., and Kurths, J. (2003). Synchronization: A Universal Concept in Nonlinear Sciences, Vol. 12. Cambridge: Cambridge University Press.

Polak, R., London, J., and Jacoby, N. (2016). Both isochronous and non-isochronous metrical subdivision afford precise and stable ensemble entrainment: a corpus study of malian jembe drumming. Front. Neurosci. 10:285. doi: $10.3389 /$ fnins.2016.00285

Ravignani, A. (2014). Chronometry for the chorusing herd: Hamilton's legacy on context-dependent acoustic signalling-a comment on Herbers (2013). Biol. Lett. 10, 20131018. doi: 10.1098/rsbl.2013.1018

Ravignani, A. (2017a). Interdisciplinary debate: agree on definitions of synchrony. Nature 545, 158. doi: 10.1038/545158c

Ravignani, A. (2017b). Visualizing and interpreting rhythmic patterns using phase space plots. Music Percept. 34, 557-568. doi: 10.1525/mp.2017.34.5.557
Ravignani, A., Bowling, D., and Kirby, S. (2014a). The psychology of biological clocks: a new framework for the evolution of rhythm. Paper Presented at the Evolution of Language: Proceedings of the 10th international conference, Singapore.

Ravignani, A., Bowling, D. L., and Fitch, W. T. (2014b). Chorusing, synchrony and the evolutionary functions of rhythm. Front. Psychol. 5:1118. doi: 10.3389/fpsyg. 2014.01118

Ravignani, A., and Cook, P. (2016). The evolutionary biology of dance without frills. Curr. Biol. 26, R878-R879. doi: 10.1016/j.cub.2016.07.076

Ravignani, A., Delgado, T., and Kirby, S. (2016a). Musical evolution in the lab exhibits rhythmic universals. Nat. Hum. Behav. 1:0007.

Ravignani, A., Fitch, W. T., Hanke, F. D., Heinrich, T., Hurgitsch, B., Kotz, S. A., et al. (2016b). What pinnipeds have to say about human speech, music, and the evolution of rhythm. Front. Neurosci. 10:274. doi: 10.3389/fnins.2016. 00274

Ravignani, A., Honing, H., and Kotz, S. A. (2017). The evolution of rhythm cognition: timing in music and speech. Front. Hum. Neurosci. 11:303. doi: $10.3389 /$ fnhum.2017.00303

Ravignani, A., and Norton, P. (2017). Measuring rhythmic complexity: a primer to quantify and compare temporal structure in speech, movement, and animal vocalizations. J. Lang. Evol. 2, 4-19. doi: 10.1093/jole/lzx002

Repp, B. H. (2005). Sensorimotor synchronization: a review of the tapping literature. Psychon. Bullet. Rev. 12, 969-992. doi: 10.3758/BF03206433

Repp, B. H., and Su, Y.-H. (2013). Sensorimotor synchronization: a review of recent research (2006-2012). Psychon. Bullet. Rev. 20, 403-452. doi: 10.3758/s13423012-0371-2

Richter, J., and Ostovar, R. (2016). "It don't mean a thing if it ain't got that Swing"an alternative concept for understanding the evolution of dance and music in human beings. Front. Hum. Neurosci. 10:485. doi: 10.3389/fnhum.2016.00485

Roach, P. (1982). On the distinction between 'stress-timed' and 'syllabletimed'languages. Linguist. Controversies 73, 79.

Rouse, A. A., Cook, P. F., Large, E. W., and Reichmuth, C. (2016). Beat keeping in a sea lion as coupled oscillation: implications for comparative understanding of human rhythm. Front. Neurosci. 10:257. doi: 10.3389/fnins.2016.00257

Ryan, M. J. (1998). Sexual selection, receiver biases, and the evolution of sex differences. Science 281, 1999-2003. doi: 10.1126/science.281.5385.1999

Schirmer, A., Meck, W. H., and Penney, T. B. (2016). The socio-temporal brain: connecting people in time. Trends Cogn. Sci. 20, 760-772. doi: 10.1016/j.tics. 2016.08.002

Schultz, B. G., O’Brien, I., Phillips, N., McFarland, D. H., Titone, D., and Palmer, C. (2016). Speech rates converge in scripted turn-taking conversations. Appl. Psycholinguist. 37, 1201-1220. doi: 10.1017/S0142716415000545

Schusterman, R. J. (1977). Temporal patterning in sea lion barking (Zalophus californianus). Behav. Biol. 20, 404-408. doi: 10.1016/S0091-6773(77)90964-6

Scott-Phillips, T. C., Kirby, S., and Ritchie, G. R. (2009). Signalling signalhood and the emergence of communication. Cognition 113, 226-233. doi: 10.1016/ j.cognition.2009.08.009

Sismondo, E. (1990). Synchronous, alternating, and phase-locked stridulation by a tropical katydid. Science 249, 55-58. doi: 10.1126/science.249.4964.55

Stivers, T., Enfield, N. J., Brown, P., Englert, C., Hayashi, M., Heinemann, T., et al. (2009). Universals and cultural variation in turn-taking in conversation. Proc. Natl. Acad. Sci. U.S.A. 106, 10587-10592. doi: 10.1073/pnas.0903616106

Strogatz, S. H. (2000). From Kuramoto to crawford: exploring the onset of synchronization in populations of coupled oscillators. Physica D 143, 1-20. doi: 10.1016/S0167-2789(00)00094-4

Strogatz, S. H. (2003). Sync: The Emerging Science of Spontaneous Order. New York City, NY: Hyperion Books.

Strogatz, S. H., and Stewart, I. (1993). Coupled oscillators and biological synchronization. Sci. Am. 269, 102-109. doi: 10.1038/scientificamerican 1293-102

Su, Y.-H. (2016a). Sensorimotor synchronization with different metrical levels of point-light dance movements. Front. Hum. Neurosci. 10:186. doi: 10.3389/ fnhum.2016.00186

Su, Y.-H. (2016b). Visual enhancement of illusory phenomenal accents in non-isochronous auditory rhythms. PLOS ONE 11:e0166880. doi: 10.1371/ journal.pone.0166880

Teie, D. (2016). A comparative analysis of the universal elements of music and the fetal environment. Front. Psychol. 7:1158. doi: 10.3389/fpsyg.2016.01158 
Tierney, A., White-Schwoch, T., MacLean, J., and Kraus, N. (2017). Individual differences in rhythmic skills: links with neural consistency and linguistic ability. J. Cogn. Neurosci. 29, 855-868. doi: 10.1162/jocn_a_01092

Tinbergen, N. (1963). On aims and methods of ethology. Z. Tierpsychol. 20, 410-433. doi: 10.1111/j.1439-0310.1963.tb01161.x

Tomasello, M. (2009). The Cultural Origins of Human Cognition. Cambridge, MA: Harvard University Press.

Toussaint, G. T. (2013). The Geometry of Musical Rhythm: What Makes a" good" Rhythm Good?. Boca Raton, FL: CRC Press.

Tuller, B., and Fowler, C. A. (1980). Some articulatory correlates of perceptual isochrony. Atten. Percept. Psychophys. 27, 277-283. doi: 10.3758/BF03206115

Ukkola, L. T., Onkamo, P., Raijas, P., Karma, K., and Järvelä, I. (2009). Musical aptitude is associated with AVPR1A-haplotypes. PLOS ONE 4:e5534. doi: 10.1371/journal.pone.0005534

Ukkola-Vuoti, L., Kanduri, C., Oikkonen, J., Buck, G., Blancher, C., Raijas, P., et al. (2013). Genome-wide copy number variation analysis in extended families and unrelated individuals characterized for musical aptitude and creativity in music. PLOS ONE 8:e56356. doi: 10.1371/journal.pone.0056356

Vorobyev, M., Osorio, D., Bennett, A. T., Marshall, N., and Cuthill, I. (1998). Tetrachromacy, oil droplets and bird plumage colours. J. Comp. Physiol. A 183, 621-633. doi: 10.1007/s003590050286

Wade, B. C. (2004). Thinking Musically: Experiencing Music, Expressing Culture. New York, NY: Oxford University Press.
Wilson, M., and Cook, P. F. (2016). Rhythmic entrainment: why humans want to, fireflies can't help it, pet birds try, and sea lions have to be bribed. Psychon. Bullet. Rev. 23, 1647-1659. doi: 10.3758/s13423-016-1013-x

Winfree, A. T. (1986). Timing of Biological Clocks. New York City, NY: Henry Holt and Company.

Wing, A. M., and Kristofferson, A. B. (1973a). Response delays and the timing of discrete motor responses. Atten. Percept. Psychophys. 14, 5-12.

Wing, A. M., and Kristofferson, A. B. (1973b). The timing of interresponse intervals. Atten. Percept. Psychophys. 13, 455-460. doi: 10.3758/BF03205802

Zornik, E., and Kelley, D. B. (2011). A neuroendocrine basis for the hierarchical control of frog courtship vocalizations. Front. Neuroendocrinol. 32:353-366. doi: 10.1016/j.yfrne.2010.12.006

Conflict of Interest Statement: The authors declare that the research was conducted in the absence of any commercial or financial relationships that could be construed as a potential conflict of interest.

Copyright (c) 2017 Ravignani and Madison. This is an open-access article distributed under the terms of the Creative Commons Attribution License (CC BY). The use, distribution or reproduction in other forums is permitted, provided the original author(s) or licensor are credited and that the original publication in this journal is cited, in accordance with accepted academic practice. No use, distribution or reproduction is permitted which does not comply with these terms. 